\title{
Hallazgos clínicos y electrocardiográficos en pacientes pediátricos con comunicación interauricular
}

\author{
Clinical and electrocardiographic findings in pediatric \\ patients with atrial septal defects
}

\author{
Dong Chin Suh', Junior Greco', Fátima Romero', Lorena Spinzi', Chantal Real', Milagros \\ Vargas-Peña', Jorge Jarolín ${ }^{1}$
}

\section{RESUMEN}

Introducción: La comunicación interauricular (CIA) es una cardiopatía congénita frecuente, cuyo diagnóstico es difícil en pacientes paucisintomáticos. Sin tratamiento, puede complicarse con insuficiencia cardiaca, arritmias e hipertensión pulmonar. El electrocardiograma puede proporcionar indicios de esta cardiopatía. Objetivo: Describir los hallazgos clínicos y electrocardiográficos de pacientes portadores de CIA. Materiales y Métodos: Trabajo retrospectivo, de pacientes menores de 18 años, evaluados cardiológicamente de enero 2008 a diciembre de 2013. Se describen las variables electrocardiográficas y semiológicas sugestivas de CIA. Resultados: Hubo 77 pacientes con CIA. Los motivos de consulta más frecuentes entre los pacientes con CIA fueron el soplo cardiaco, el síndrome de Down y la disnea. En el examen físico de los pacientes con CIA: soplo sistólico en $69 \%$ y R2 desdoblado en 56\%. En el electrocardiograma, los pacientes tuvieron QRS de $79 \mathrm{~ms}$, eje eléctrico desviado hacia la derecha, BIRD y notch. Los criterios de sobrecarga ventricular derecha (SVD) más frecuentes fueron la onda S profunda en V6 y la onda R alta en V1. La auscultación de un R2 desdoblado en el examen físico, así como la SVD y el patrón notch fueron los hallazgos más frecuentes en pacientes con CIA. Conclusión: el electrocardiograma constituye una herramienta válida en la evaluación de pacientes con sospecha de CIA pero precisa de la combinación de hallazgos clínicos para llegar al diagnóstico.

Palabras claves: comunicación interauricular, electrocardiograma, bloqueo incompleto de rama derecha, notch.

\section{ABSTRACT}

Introduction: Atrial Septal Defect (ASD) is a frequent congenital heart disease, the diagnosis of which is difficult in paucisymptomatic patients. Without treatment, it can lead to heart failure, arrhythmias and pulmonary hypertension. The electrocardiogram may provide evidence of this condition. Objective: To describe the clinical and electrocardiographic findings of patients with ASD. Materials and Methods: This was a retrospective study of patients under age 18 who underwent cardiac evaluation from January 2008 to December 2013. The electrocardiographic and clinical finding variables suggestive of ASD are described. Results: There were 77 patients with ASD. The most frequent reasons for consultation among patients with ASD were heart murmur, Down syndrome and dyspnea. Findings on physical examination of patients with ASD were: systolic murmur in $69 \%$ and S2 split in $56 \%$. On the electrocardiogram, the patients had QRS of $79 \mathrm{~ms}$, electric axis deviated to the right, Incomplete Right Bundle Branch and notch pattern. The most frequent right ventricular volume overload (RVVO) criteria were the deep $S$ wave in V6 and the high R wave in V1. Auscultation of a split S2 on the physical examination, as well as the RVVO and the notch pattern were the most frequent findings in patients with ASD. Conclusion: the electrocardiogram is a valid tool in the evaluation of patients with suspected ASD but requires the combination of clinical findings to reach the diagnosis.

Keywords: Atrial septal defect, electrocardiogram, incomplete right bundle branch block, notch.

\footnotetext{
${ }^{1}$ Departamento de Cardiología Pediátrica, Hospital de Clínicas, Facultad de Ciencias Médicas, Universidad Nacional de Asunción. San Lorenzo, Paraguay.

Correspondencia: Milagros Vargas Peña Correo: milavargaspena@gmail.com

Conflicto de Interés: Los autores declaran no poseer conflicto de interés

Recibido: 16/10/2019 Aceptado: 25/10/2019

Doi: https://doi.org/10.31698/ped.46032019005
}

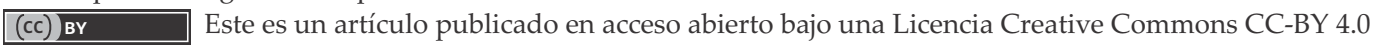




\section{INTRODUCCIÓN}

La comunicación interauricular (CIA) o defecto del septo interatrial representa el tercer tipo más frecuente de cardiopatía congénita acianótica ${ }^{(1)}$. Las principales variedades anatómicas son las de seno venoso, ostium secundum, ostium primum y el foramen oval permeable. La historia natural y las manifestaciones clínicas dependen del tipo anatómico y el tamaño del defecto; en su mayoría los pacientes son poco sintomáticos, incluso con lesiones hemodinámicamente significativas. Es así que un defecto no detectado en etapas tempranas, puede debutar con signos y síntomas de insuficiencia cardiaca, hipertensión pulmonar y arritmias en la adultez ${ }^{(2)}$.

Clínicamente, estos defectos pueden ser sospechados en casos de moderada a severa repercusión, cuando los pacientes presentan cuadros respiratorios recurrentes, escasa ganancia ponderal o hallazgos en la auscultación cardíaca (segundo ruido desdoblado o soplo sistólico pulmonar $)^{(3)}$. Dentro de las herramientas paraclínicas, el electrocardiograma se considera como un elemento que puede llevar a la sospecha de un defecto del septo interatrial, cuando presenta signos de sobrecarga de cavidades derechas o alteraciones en la morfología del QRS o la onda $\mathrm{T}^{(4)}$.

Este trabajo tiene como objetivo describir los hallazgos del electrocardiograma en conjunción con hallazgos clínicos, como herramienta diagnóstica en pacientes con CIA.

\section{MATERIALES Y MÉTODOS}

Estudio retrospectivo, de pacientes menores de 18 años, de ambos sexos, con diagnóstico confirmado de CIA por ecocardiografía, que acudieron a consultorio externo del departamento de Cardiología Pediátrica del Hospital de Clínicas (San Lorenzo, Paraguay) durante el período comprendido entre enero de 2008 y diciembre de 2013. Fueron excluidos pacientes con defecto del septo interauricular asociado a otros defectos cardiacos.

Las fichas clínicas proporcionaron los datos clínicos y demográficos, incluyendo los hallazgos físicos sugestivos de CIA como ser la presencia de soplo o desdoblamiento fijo del segundo ruido cardiaco. El tipo y el tamaño del defecto fueron obtenidos del informe ecocardiográfico.

En el ECG, los patrones sugestivos fueron: la presencia de sobrecarga de cavidades derechas, variaciones del intervalo PR y la duración del QRS, disturbios de conducción intraventricular, la presencia de melladura o "notch" en las derivaciones inferiores y alteraciones de la repolarización ventricular.

El patrón crochetage o notch fue definido como la presencia de una melladura en la onda $\mathrm{R}$ o la onda $S$ en las derivaciones inferiores (DII, DIII y a VF).

Se ha definido como BIRD la presencia de un complejo QRS trifásico en la derivación V1, con duración menor de 120 ms y patrón $\mathrm{rSr}^{\prime}$, o rsR'. Cualquier otro patrón, que tuviera alguna melladura en el complejo QRS fue clasificado como disturbio inespecífico de conducción intraventricular. Los ejemplos electrocardiográficos se muestran en las Figuras 1,2 y 3 .

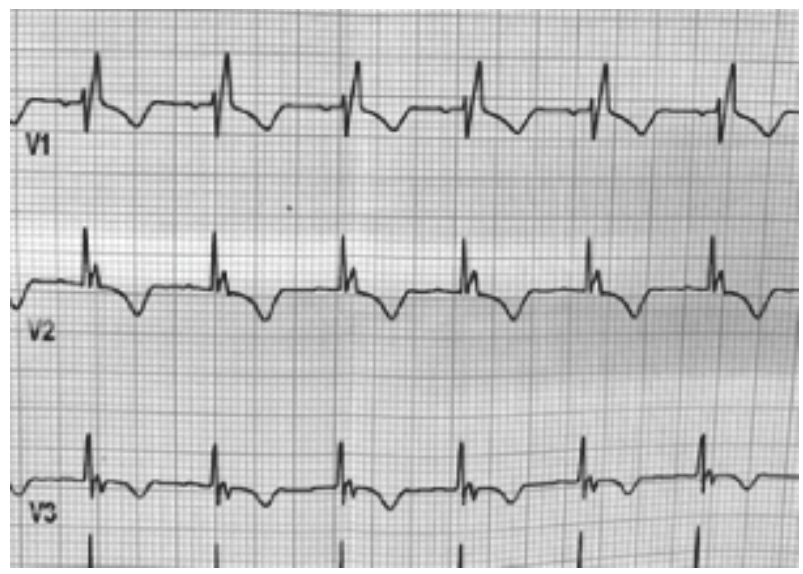

Figura 1. Bloqueo incompleto de rama derecha. 


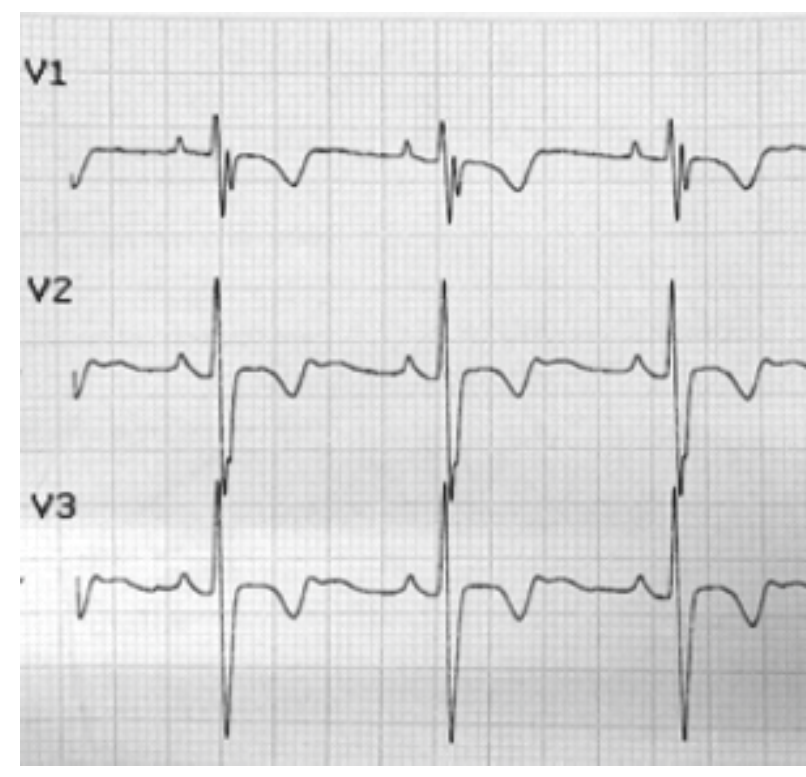

Figura 2. Disturbio inespecífico de la conducción ventricular.

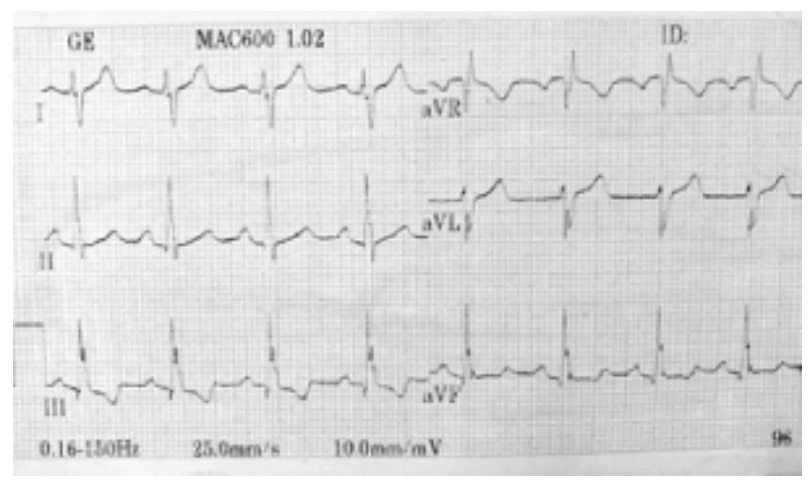

Figura 3. Patrón notch en derivaciones inferiores.

Por su naturaleza retrospectiva y manteniendo el anonimato de los pacientes, no hubo requerimiento de consentimiento informado.

\section{RESULTADOS}

Fueron analizados 77 pacientes con CIA (48 mujeres, 29 varones). Las características demográficas de ambos grupos se detallan en la Tabla 1.

Tabla 1. Características demográficas de pacientes con CIA.

\begin{tabular}{lc}
\hline & CIA (n: 77) \\
\hline Edad (años) & $5.25 \pm 4.8$ \\
Mujeres (\%) & $48(62)$ \\
Peso kg (promedio \pm DE) & $20.5 \pm 15.4$ \\
Talla cm & $105.3 \pm 35.4$ \\
\hline
\end{tabular}

El hallazgo de soplo cardiaco en el examen pediátrico fue el motivo principal de remisión, en un $42 \%$ de los pacientes con CIA. Los demás síntomas que llevaron a la sospecha de cardiopatía fueron el cansancio al mamar en los lactantes y la disnea de esfuerzo en los niños mayores (13\%). En 11 pacientes (14\%) con CIA, la evaluación cardiológica fue debida a la presencia de síndrome de Down. Los demás motivos de remisión se detallan en la Tabla 2.

Los tipos de CIA fueron: ostium secundum 63 pacientes $(82 \%)$, foramen oval $9(11,5 \%)$, ostium primum $2(2.5 \%)$ y seno venoso $1(1,2 \%)$. El tamaño promedio del defecto fue de $12.6 \pm 7.12 \mathrm{~mm}$, medido por ecocardiografía transtorácica.

El examen físico reveló soplo cardíaco en 53 de ellos (69\%); el segundo ruido cardiaco (R2) estaba desdoblado en 43 pacientes (56\%). En 20 casos (26\%), la auscultación se consignó como normal.

Respecto a los hallazgos electrocardiográficos, solo 14 pacientes (18\%) portadores de CIA tuvieron un ECG totalmente normal. El criterio de sobrecarga ventricular derecha más frecuente fue la amplitud anormal de la onda S en la derivación V6, presente en 17 de ellos (22\%). El siguiente criterio, presente en 9 casos (11.7\%), fue la amplitud aumentada de la onda R en V1. Tuvieron uno o más criterios de sobrecarga ventricular derecha 23 pacientes $(30 \%)$ El intervalo PR estaba prolongado en 2 casos. La sobrecarga atrial derecha (aumento de la amplitud de la onda P) estuvo presente en 9 casos. Otros hallazgos fueron el BIRD (46\%), el eje QRS desviado a la derecha y la sobrecarga atrial derecha. El ritmo fue sinusal en todos los casos. Ver Tablas 3 y 4. 
Tabla 2. Motivos de consulta y remisión al consultorio de Cardiología Pediátrica de los portadores de CIA.

\begin{tabular}{lcc}
\hline Motivo de consulta/remisión & Número de pacientes & (Porcentaje) \\
\hline Soplo cardiaco & 33 & $43 \%$ \\
Síndrome de Down & 11 & $14 \%$ \\
Disnea, cansancio al mamar & 10 & $13 \%$ \\
Antecedente de prematurez & 4 & $5.2 \%$ \\
Cuadros respiratorios recurrentes & 3 & $4 \%$ \\
Hallazgo casual de la CIA & 3 & $4 \%$ \\
Arritmia supraventricular & 2 & $2.6 \%$ \\
Tuberculosis ECG alterado & 2 & $2.6 \%$ \\
Cardiomegalia & 1 & $1.3 \%$ \\
(Radiografía de tórax) & 1 & $1.3 \%$ \\
Sin datos & 7 & $9 \%$ \\
\hline Total & 77 & $\mathbf{1 0 0} \%$ \\
\hline
\end{tabular}

Tabla 3. Hallazgos electrocardiográficos de pacientes con CIA.

\begin{tabular}{lc}
\hline & CIA (n: 77) \\
\hline Intervalo PR (ms) & $120.7 \pm 30$ \\
Duración QRS (ms) & $79 \pm 11$ \\
Eje QRS & $74.8 \pm 47.9$ \\
Casos con BIRD (\%) & $36(46)$ \\
Casos con Notch (\%) & $20(26)$ \\
BIRD + Notch & $12(15)$ \\
\hline
\end{tabular}

Tabla 4. Criterios de sobrecarga de cavidades derechas en pacientes con CIA. N:77.

\begin{tabular}{lcc}
\hline Criterio & Número & (Porcentaje) \\
\hline Onda s profunda en V6 (Sv6) & 14 & $18 \%$ \\
Onda R alta en V1 (Rv1) & 2 & $2.5 \%$ \\
Presentes SV6 y Rv1 & 8 & $9 \%$ \\
Sobrecarga atrial derecha & 9 & $12 \%$ \\
\hline
\end{tabular}

\section{DISCUSIÓN}

La CIA es una de las cardiopatías congénitas más frecuentes $^{(2)}$. La sintomatología es bastante inespecífica y variable, por lo que el diagnóstico clínico es dificultoso. Además, los signos semiológicos clásicos pueden pasar desapercibidos en los casos en que el examen del aparato cardiovascular no es preciso o exhaustivo; por ello, el electrocardiograma constituye una herramienta útil en la evaluación, ya que puede mostrar unos hallazgos sugestivos del defecto.

En el electrocardiograma de pacientes con CIA, hemos encontrado una leve desviación de eje del
QRS a la derecha, un leve aumento en la duración del QRS, así como casos con BIRD (46\%) y patrón notch en derivaciones inferiores (26\%). En el trabajo de Bayar et al., encontraron una prevalencia de BIRD de $56 \%$ y de notch de $57 \%$ en una población con $\mathrm{CIA}^{(4)}$.

Esta cardiopatía congénita predomina en mujeres, y está asociada habitualmente a una contextura física descrita como "grácil", es decir, pacientes con talla y peso bajos, sin signos de desnutrición ${ }^{(5)}$. Los motivos de consulta más frecuentes que llevaron al diagnóstico en nuestra serie fueron la presencia de soplo cardiaco, disnea de esfuerzo y la evaluación 
por presencia de síndrome de Down.

El ecocardiograma transtorácico es el principal método de diagnóstico, pero existen en la literatura reportes del uso del electrocardiograma como medio de cribado. Los signos electrocardiográficos utilizados son variados, desde las alteraciones propias de la sobrecarga de cavidades derechas, los disturbios de conducción intraventricular y la repolarización ventricular. Entre los disturbios de la conducción intraventricular más estudiados, se pueden mencionar principalmente al bloqueo de rama derecha y al patrón "notch" de derivaciones inferiores ${ }^{(6-9)}$.

La presencia de una onda $S$ por encima de los valores normales en la derivación V6, fue el criterio de sobrecarga ventricular derecha más frecuentemente hallado en esta serie, seguido del hallazgo de la onda $\mathrm{R}$ en V1 mayor al valor normal. Existieron casos con presencia de al menos dos criterios de sobrecarga ventricular derecha. Otros hallazgos también fueron el BIRD, el eje QRS desviado a la derecha y la sobrecarga atrial derecha, estando presentes algunos de ellos en forma combinada. Arrington et al. obtuvieron resultados similares, encontrando que el criterio más frecuente de sobrecarga ventricular derecha en pacientes con CIA fue la onda S profunda en V6, presente en $48 \%$ de los $\operatorname{casos}^{(7)}$.

En un estudio reciente de Lei Shen et al., la aparición de notch en las tres derivaciones inferiores dio una sensibilidad de $28 \%$ y una especificidad de $94.5 \%$ en el diagnóstico de CIA; la presencia de notch en una derivación más patrón de BIRD dio una sensibilidad de $15 \%$ y una especificidad de $98.8 \%$.No se conoce la causa de este patrón electrocardiográfico trifásico en las derivaciones frontales inferiores, pero se ha asociado a defectos interauriculares de mayor tamaño o repercusión hemodinámica; también se ha constatado su desaparición en pacientes posoperados. En esta serie constatamos la presencia de patrón notch en el $26 \%$ de los pacientes con diagnóstico de CIA, así como la asociación de la presencia de BIRD con patrón notch en el electrocardiograma en $15 \%$ de los pacientes. Teniendo en cuenta que la presencia de dicho patrón podría asociarse a defectos de gran repercusión, podríamos atribuir el bajo porcentaje de hallazgo del mismo en esta serie a que fueran incluidos pacientes con defectos de menor repercusión, siendo esta una hipótesis que pudiera ser motivo de investigaciones posteriores.

El complejo QRS a menudo tiene patrones rSr'o rsR', que se consideran como variantes normales. Son descritos habitualmente como bloqueo incompleto de rama derecha si la duración del complejo QRS es menor de $120 \mathrm{~ms}$. Se considera una variante normal, producto de la despolarización de las regiones basales ventriculares; con los cambios vectorcardiográficos que aparecen en el niño hasta su maduración, pueden aparecer patrones polifásicos o atípicos del complejo QRS en V1, habitualmente informados como disturbios inespecíficos de la conducción intraventricular. Otras causas posibles de aparición de estas morfologías, tanto de los patrones trifásicos o los disturbios inespecíficos de la conducción intraventricular, son la colocación inexacta de los electrodos precordiales V1 y V2, generalmente en el segundo espacio intercostal y el pectus excavatum. No se deben olvidar las causas patológicas graves de patrones rsr' en V1, como el síndrome de Brugada y la displasia arritmogénica del ventrículo derecho ${ }^{(9)}$.

El BIRD es un patrón frecuente en los pacientes con CIA, se ha considerado como resultado de un engrosamiento desproporcionado del tracto de salida del ventrículo derecho, que es la última porción del ventrículo en despolarizarse. Sin embargo, en un estudio electrofisiológico de Sung et al. no se observó diferencia entre pacientes con CIA y personas sanas en cuanto a la velocidad de conducción en el tracto de salida del ventrículo derecho ${ }^{(10)}$. Puede estar presente hasta en más del $90 \%$ de los pacientes con CIA y hasta un $10 \%$ de pacientes sin cardiopatía. El desafío clínico se encuentra al tener un paciente con hallazgo casual de BIRD en un control rutinario, teniendo que decidir si amerita la realización de un ecocardiograma. En base a los hallazgos de este estudio creemos que, al asociar algunos datos electrocardiográficos como los criterios de sobrecarga derecha y el notch, además de los hallazgos semiológicos (R2 con desdoblamiento fijo, soplo sistólico), es altamente probable encontrar un defecto atrial en un paciente pediátrico.

Las alteraciones de la repolarización ventricular 
como la persistencia de la onda $\mathrm{T}$ positiva en $\mathrm{V} 1$ y V2 en pacientes mayores de 3 días de vida, como dato de sobrecarga ventricular derecha, y el hallazgo de una onda $\mathrm{T}$ negativa aislada en derivaciones precordiales podrían estar presentes en el electrocardiograma de pacientes con CIA ${ }^{(11)}$. En esta revisión no hemos encontrado ningún caso de sobrecarga ventricular derecha con onda $\mathrm{T}$ positiva en V1. Respecto a la onda T negativa aislada, no la hemos considerado en nuestro análisis debido a que la repolarización ventricular en el niño tiene amplias variaciones normales del eje de la onda $\mathrm{T}$ en las derivaciones precordiales.

Consideramos limitaciones de este trabajo las inherentes a su naturaleza retrospectiva y al limitado número de pacientes analizados en esta serie.

\section{REFERENCIAS BIBLIOGRÁFICAS}

1. Qu Y, Liu X, Zhuang J, Chen G, Mai J, Guo X, et al. Incidence of congenital heart disease: The 9-year experience of the Guangdong Registry of congenital heart disease, China. PLoS ONE. 2016; 11(7):e0159257. DOI: https://doi.org/10.1371/journal.pone.0159257

2. Zvaigzne CG, Howarth AG, Patton DJ. Atrial shunts: presentation, investigation, and management, including recent advances in magnetic resonance imaging. Cardiology in the Young. 2014; 24(3):403-416. DOI: https://doi.org/10.1017/S1047951113002321

3. Geva T, Martins JD, Wald RM. Atrial septal defects. The Lancet. 2014; 383(9932):1921-1932. DOI: https://doi.org/10.1016/S0140-6736(13)62145-5

4. Bayar N, Arsian S, Koklu E, Cagirci G, Cay S, Erkal Z, et al. The importance of electrocardiographic findings in the diagnosis of atrial septal defect. Kardiol Pol. 2015; 73(5):331-336. DOI: https://doi.org/10.5603/KP.a2014.0240

5. English RF, Anderson RH, Ettedgui JA. Interatrial communications. En: Anderson RH, Baker EJ, Penny D, editores. Paediatric Cardiology. Philadelphia: Churchil Livingstone; 2010. p. 523-546.

6. Schiller O. The por performance of RSR' pattern on electrocardiogram as a screening test for large atrial septal defects in children. J Pediatr. 2013; 162:308-312. DOI: https://doi.org/10.1016/j.jpeds.2012.07.017

\section{CONCLUSIÓN}

Teniendo en cuenta la asociación de SVD, BIRD y patrón notch en pacientes con diagnóstico de CIA, consideramos que el electrocardiograma constituye una herramienta válida en la evaluación de pacientes con sospecha de CIA, pero precisa de la combinación de hallazgos clínicos para llegar al diagnóstico.

Debido a que constituye un estudio de bajo costo y de mayor accesibilidad constituye la primera línea en el diagnóstico de esta cardiopatía, siendo sin embargo indispensable la realización del ecocardiograma para confirmar el diagnóstico.

7. Arrington CB, Tani LY, Minich LL, Bradley DJ. An assesment of the electrocardiogram as a screening test for large atrial septal defects in children. Journal of Electrocardiology. 2007; 40:484-488. DOI: https://doi.org/10.1016/j.jelectrocard.2007.06.001

8. Shen L, Liu J, Li JK et al. The significance of crochetage on the $\mathrm{R}$ wave of an electrocardiogram for the early diagnosis of pediatric secundum atrial septal defect. Pediatric Cardiology. 2018; 39(5):1031-1035. DOI: https://doi.org/10.1007/s00246-018-1857-0

9. Baranchuk A, Enriquez A, García-Niebla J, Bayés-Genís A, Villuendas R, Bayés de Luna A. Differential diagnosis of rSr' pattern in leads V1-V2. Comprehensive review and proposed algorithm. Ann Noninvasive Electrocardiol. 2015; 20(1):7-17. DOI: https://doi.org/10.1111/anec.12241

10. Sung RJ, Tamer DM, Agha AS, Castellanos A, Myerburg RJ, Gelband H. Etiology of the electrocardiographic pattern of "incomplete right bundle branch block" in atrial septal defect: an electrophysiologic study. J Pediatr. 1975; 87(6 Pt 2):1182-6. DOI: https://doi.org/10.1016/s0022-3476(75)80133-8

11. Somura J, Nakagawa $M$, Ukiami $M$, Sagawa $H$, Furukawa O, Hoshino S, et al. Relationship between electrocardiographic signs and shunt volumen in atrial septal defect. Pediatrics International. 2015; 57: 535-540. DOI: https://doi.org/10.1111/ped.12569 